Instructions for authors, subscriptions and further details:

http://qre.hipatiapress.com

\title{
El Agar.io Programado en la Enseñanza Superior: Fundamentos de la Dinámica del Superrendimiento y la Superproducción
}

Gustavo González-Calvo ${ }^{1}$

1) Departamento de Didáctica de la Expresión Musical, Plástica y Corporal, Universidad de Valladolid, España.

Date of publication: June $28^{\text {th }}, 2020$

Edition period: June 2020 - October 2020

To cite this article: González-Calvo, G. (2020). El Agar.io Programado en la Enseñanza Superior: Fundamentos de la Dinámica del Superrendimiento y la Superproducción. Qualitative Research in Education, 9(2), 160-187. doi:10.17583/qre.2020.5412

To link this article: $h t t p: / / d x . d o i . o r g / 10.17583 / q r e .2020 .5412$

\section{PLEASE SCROLL DOWN FOR ARTICLE}

The terms and conditions of use are related to the Open Journal System and to Creative Commons Attribution License (CC-BY). 


\section{Programmed Agar.io in Higher Education: Fundaments of the Dynamics of Overproduction and Superproduction}

Gustavo González-Calvo

University of Valladolid

(Received: 12 April 2020; Accepted: 15 June 2020; Published: 28 June 2020)

\section{Abstract}

This article explores the experiences and particularities faced by partial/full time-lecturers in a precarious situation who opt, without apparent success, for a full time job within the professional system of higher education. Following a narrative methodology, it explores the way in which teachers (eight women and six men, all of them university partial or full time lecturers in different areas) construct and legitimize certain conceptions about the professional sphere in the university that have obvious repercussions on their personal and professional identities. The results, organized around a narrative that takes whose reference is the daily life of a teacher in precarious conditions, reflect the stress to which teachers are subjected as part of the relentless programs of university evaluation and accreditation, the distancing of good teaching in favour of demonstrable quality as an investigator and the possibility of suffering from teacher burnout syndrome no sooner than having commenced a professional career.

Keywords: Precariat, narratives, neoliberalism, university career 


\section{El Agar.io Programado en la Enseñanza Superior: Fundamentos de la Dinámica del Superrendimiento y la Superproducción}

Gustavo González-Calvo

University of Valladolid

(Recibido: 12 de abril de 2020; Aceptado: 15 de junio de 2020; Publicado: 28 de junio de 2020)

\section{Resumen}

En este artículo se exploran las experiencias y particularidades a las que se enfrentan los profesores a tiempo parcial o completo en situación precaria que optan, sin éxito aparente, por un trabajo a tiempo completo dentro del sistema profesional de la enseñanza superior. Siguiendo una metodología narrativa, explora la forma en que los profesores (ocho mujeres y seis hombres, todos ellos docentes universitarios a tiempo parcial o completo en diferentes áreas) construyen y legitiman ciertas concepciones sobre la esfera profesional en la universidad que tienen evidentes repercusiones en su identidad personal y profesional. Los resultados, organizados en torno a un relato que toma como referencia la vida cotidiana de un profesor en condiciones precarias, reflejan el estrés al que se ven sometidos los docentes en el marco de los incesantes programas de evaluación y acreditación universitaria, el distanciamiento de la buena enseñanza en favor de una calidad demostrable como investigador y la posibilidad de sufrir el síndrome de agotamiento del profesorado nada más iniciar su carrera profesional.

Palabras clave: Precariedad, narrativas, neoliberalismo, carrera profesional en la universidad 
E

1 videojuego Agar.io, popularizado en diferentes series de televisión, es un sencillo juego en el que cada jugador se representa por una pequeña esfera. El objetivo es tratar de llegar a ser lo más grande que cada uno pueda, para lo que ha de "comer" a otras esferas y evitar ser devorado por otras mayores. Es la misma imagen del sistema neoliberal y capitalista actual: la jungla, la supervivencia, el "solo puede triunfar uno", el "todos contra todos" y, en última instancia, el "todos contra nosotros mismos". Hay que permanecer en continuo movimiento: quien no avanza, quien duda, es devorado. Sobrevivir o perecer, terrible metáfora del sistema neoliberal universitario de hoy día.

No en vano, a día de hoy los profesores universitarios -en mayor medida los que trabajan en condiciones precarias- se ven sometidos a una innegable presión para cumplir los requisitos del sistema, asociados a términos empresariales y capitalistas como excelencia, productividad y superación de rigurosas evaluaciones (González-Calvo, 2020; González-Calvo \& AriasCarballal, 2018). De este modo, el mundo universitario se parece cada vez más a una gran empresa privada y ligada a la producción de bienes públicos y técnicas de gestión tradicionalmente vinculadas al sector privado y empresarial (e.g. Chiapello, 2017; Lorenz, 2012; Luna, 2015), todo bajo la convicción de que dichas técnicas son imprescindibles para alcanzar el desarrollo económico y tecnológico y el control social que toda economía moderna necesita (Deem et al., 2007). De este modo, es fácil comprender cómo el sistema universitario está en manos de un modelo hegemónico que condiciona muchas de sus prácticas educativas que le lleva a incorporar, en sus políticas, fórmulas para recompensar y estimular la productividad científica entre el profesorado (Chiapello, 2017).

En todo caso, a día de hoy todavía podemos hablar de la coexistencia de dos frentes claramente opuestos en la profesionalización docente: uno que defiende la idea de que reforzar la profesión implica tecnificación, burocracia, elitismo y un férreo control y vigilancia del profesor universitario; y otro que, al contrario, pretende unas condiciones contextualizadas, críticas y democráticas que permitan avanzar en el desarrollo de una nueva cultura profesional para una educación de calidad (González-Calvo, 2020).

El objetivo principal del artículo es conocer la realidad empírica que supone la aplicación de políticas neoliberales en el trabajo docente 
universitario en un contexto local como es el español. Para ello, en un intento por comprender mejor la forma en que percibimos y conocemos el mundo, a los demás y a nosotros mismos, nuestro texto emplea una metodología narrativa con la intención de generar un diálogo entre profesionales del ámbito de la educación y legisladores educativos en torno a la situación delicada que atraviesa el sistema universitario.

Para ello, primero describimos el marco teórico empleado, centrado en las características de la universidad neoliberal y el capitalismo académico. La siguiente sección explica nuestras decisiones metodológicas. El apartado de resultados y discusión, abordados de manera conjunta, persigue una mayor comprensión de los actores participantes en el estudio, así como del trabajo y las implicaciones que tiene para ellos desarrollar una carrera profesional en la universidad. La sección de resultados toma la forma de una narrativa, mostrando el que puede ser un día normal en la vida de un profesor universitario que trabaja bajo condiciones precarias. El artículo concluye cuestionando la neutralidad política y la idoneidad de las prácticas neoliberales aplicadas al mundo universitario que tienen por objeto garantizar la eficacia y la calidad del sistema, poniendo de manifiesto los nocivos efectos colaterales que estas políticas pueden tener para el profesorado que pretende hacer carrera profesional dentro de la enseñanza superior.

\section{La Carrera Profesional Universitaria en la era Neoliberal: Entusiasmos y (Des)Ilusiones}

De acuerdo con Apple (2006), el neoliberalismo es el paradigma político y económico que define específicamente la época en que vivimos, algo que requiere: la construcción de nuevas relaciones entre las diferentes formas de poder y autoridad, ampliando el alcance de la empresa; que el mundo sea más "amigable para los negocios"; y que disminuya el margen de restricción, regulación y control de las autoridades públicas sobre el poder privado (Apple, 2006; Clarke, 2008).

Dentro de la lógica neoliberal, el ser humano, considerado "capital humano" primero y, después, "empresa de sí mismo" (Laval \& Dardot, 2018), es el primer y único responsable de todo lo que le sucede; si fracasa, es a consecuencia de una vida mal administrada. Como explica Saidel (2016), 
"el sujeto neoliberal debe aprender a vivir peligrosamente, debe conocer el temor de quien arriesga, hasta el punto de ponerse paranoico para sobrevivir, aunque el resultado más probable sea la depresión" (p. 139). No en vano, las políticas neoliberales generan miedo e inseguridad. El neoliberalismo individualiza al hombre convirtiéndolo en un aislado empresario de sí mismo. La individualización que acompaña a la pérdida de solidaridad y a la competencia total, característicos entre el profesorado universitario de hoy día, provoca miedo, y el miedo incrementa la productividad (Han, 2018).

En el caso de España, como en tantos otros, el capitalismo y el neoliberalismo son los ejes vertebradores de las leyes educativas que, desde hace años, vienen dándose el relevo (e.g. Díez Gutiérrez, 2018; GonzálezCalvo, 2020; González-Calvo \& Arias-Carballal, 2018). Las políticas educativas de la educación superior, al igual que sucede en otros países, llevan a una mutación de la explotación por parte del empleador a una autoexplotación autónoma y voluntaria por parte del trabajador, en este caso, el profesor universitario. Así, el profesor que pretende hacerse un hueco en el sistema y desarrollar carrera profesional dentro de la universidad termina por convertirse en un animal laborans verdugo y víctima de sí mismo; esta explotación de sí mismo es más eficiente que la ajena, pues lleva implícita la idea de libertad (Han, 2015, 2018). Bajo la influencia de los discursos neoliberales, los individuos pueden estar convencidos de que están dando forma a sus propias condiciones de vida; sin embargo, esto es el resultado de una técnica encubierta utilizada para gobernar a los individuos persuadiéndolos para que den sentido a sus vidas como si fuera el resultado de sus propias elecciones (Han, 2018).

El sistema selectivo del profesorado universitario, enmarcado por estos preceptos neoliberales, exalta el interés propio, la competitividad, el triunfo frente a todo lo demás y, en ocasiones, las malas prácticas (González-Calvo \& Arias-Carballal, 2018). Se pretende que la persona se someta interiormente, que trabaje continuamente con el objetivo de rendir cada vez más, de conseguir una permanente mejora de sí misma (Han, 2018; Laval \& Dardot, 2018). Así, el sistema selectivo está en condiciones de integrar al profesorado en un cuerpo más flexible, dinámico y precario que adopta diferentes nombres y figuras contractuales antes de lograr una titularidad como profesor universitario, lo que deviene en una continua contractualización del profesional (desde las becas de investigación, pasando 
por la figura más precaria de profesor asociado, profesor ayudante doctor, profesor contratado doctor en sus distintas formas, etc.). Así las cosas, de acuerdo con la estadística "Datos y Cifras del Sistema Universitario Español 2018-2019", durante el curso 2016-2017 el 43,3\% del personal docente e investigador contratado en las universidades españolas fueron profesores asociados, la categoría más precaria y menos remunerada (véase figura 1).

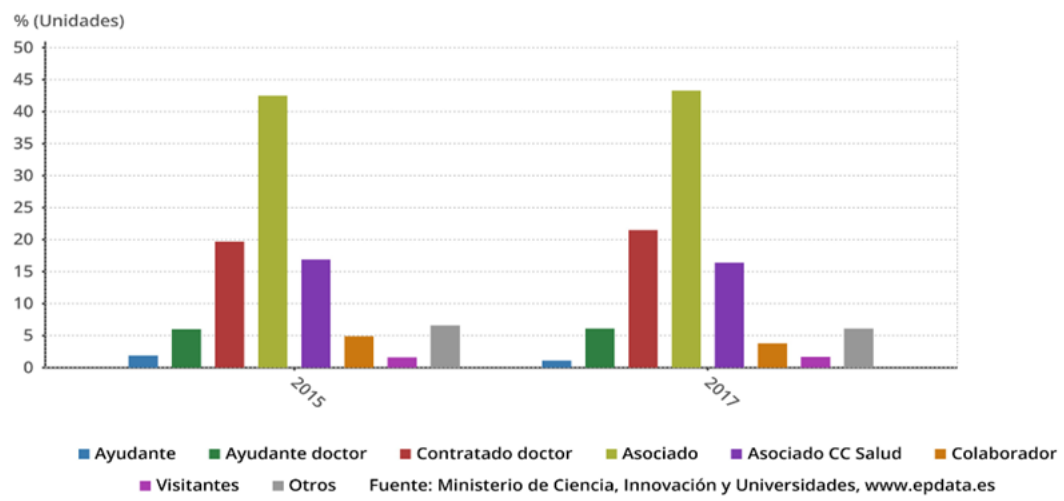

Figura 1. Personal docente e investigador en las universidades españolas según el tipo de contrato ${ }^{2}$.

La continua revalidación de la relación laboral entre empleado y universidad (Chomsky, 2013) crea las condiciones necesarias para dotar al sistema de mayor flexibilidad, eficiencia y rentabilidad, a costa de las condiciones personales y profesionales del profesorado. No en vano, la presión interna a que se ven sometidos puede llevarles a desarrollar rasgos autoagresivos, de falta de confianza en sus propias posibilidades y sentimientos de incompetencia. Viéndose forzados a aportar rendimientos, en cierto modo se asfixian a sí mismos, desarrollando miedos a quedarse al margen, a equivocarse, a fallar, a no responder a las exigencias propias (Han, 2018).

La ansiedad, la depresión, el desencanto con la profesión, el estrés laboral, son características propias de la era neoliberal, estrechamente vinculadas con la precariedad en que han de vivir el profesorado (González-Calvo \& AriasCarballal, 2018; Lorenz, 2012; Standing, 2011). El sistema, lejos de resolver la inseguridad del trabajador, alienta la competitividad, la incertidumbre y la 
precariedad. El profesor universitario, ferozmente competitivo y codicioso en tanto que sus antojos no conocen límites, vive en un estado de inquieto y permanentemente insatisfecho deseo (Bauman, 2017). Se encuentra obligado a superar su rendimiento, sus resultados, condenado a autosuperarse permanentemente, nunca está en equilibrio y nunca debe estarlo (Laval \& Dardot, 2018).

Con todo ello, el profesorado termina por caer en una perversa dinámica en torno a la idea de calidad que resulta, fundamentalmente, en un incremento de la intensidad del trabajo con la que la universidad suple las carencias que ha provocado mediante los recortes presupuestarios, los empleos precarios y el incremento de la burocracia en la profesión (e.g. Archer, 2008; Chiapello, 2017; González-Calvo \& Arias-Carballal, 2018).

\section{Metodología}

El presente estudio emplea el análisis narrativo para responder a las preguntas de la investigación. Desde los años 80 del siglo pasado, los estudiosos han comenzado a apreciar realmente la forma en que las personas estructuran sus experiencias a través de las historias que cuentan, entendiendo que una persona es esencialmente un animal que cuenta historias (Frank, 2004; Smith \& Sparkes, 2009). Smith y Sparkes (2009) explican que la narrativa, como método de investigación, es un género complejo que debe contener ciertos elementos: (a) un punto de partida; (b) protagonistas; y (c) la capacidad de conectar sucesos secuencialmente en el tiempo y el espacio para dar una explicación o consecuencia a la trama. El uso de historias para dar sentido a las experiencias está bien establecido dentro del ámbito de la investigación cualitativa (Riessman, 2008) y, además, ayuda a comprender el tejido relacional y cultural de las vidas humanas (Frank, 2010).

Los criterios que se han seguido para juzgar la calidad y la relevancia de la investigación responden a los siguientes interrogantes: la historia que se presenta, ¿evoca respuestas por parte del lector?; ¿hay algo que merezca la pena ser aprendido de la historia?; ¿abre la posibilidad de diálogo, colaboración y relación entre diferentes profesionales del ámbito de la educación superior?; ¿ayuda a plantear un cambio en las instituciones universitarias que mejore la calidad de vida de los profesionales que trabajan en ellas?; y, por último, ¿nos lleva a pensar en las consecuencias, los valores 
y los dilemas morales a que se enfrentan algunos de los profesionales a los que aquí se da voz?

En la siguiente figura (véase figura 2) se detalla el proceso de investigación llevado a cabo:

\begin{tabular}{|c|c|}
\hline & $\begin{array}{l}\text { - Diseño del proyecto } \\
\text { - Revisión teórica de la literatura y selección de los temas más } \\
\text { relevantes }\end{array}$ \\
\hline & $\begin{array}{l}\text { - Entrevistas semiestructuradas acerca del tema de estudio, tanto al } \\
\text { comienzo como al finalizar el curso escolar 2018/2019 }\end{array}$ \\
\hline & $\begin{array}{l}\text { - Análisis narrativo de los datos e identificación de los temas y } \\
\text { categorías de estudio } \\
\text { - Codificación y comparación constante de los datos }\end{array}$ \\
\hline & $\begin{array}{l}\text { - Organizacion y construcción de la narrativa a partir de las categorías y } \\
\text { los datos obtenidos en las entrevistas }\end{array}$ \\
\hline
\end{tabular}

Figura 2. Proceso de investigación llevado a cabo

\section{Participantes}

Ocho mujeres y seis hombres participaron en este estudio, todos ellos profesores universitarios en diferentes áreas (Pedagogía, Educación, Psicología, Historia y Ciencias de la Salud). De edades comprendidas entre los 32 y los 43 años, ocho de ellos se encuentran trabajando a tiempo parcial en la universidad como profesores asociados (compaginando su trabajo con otro de carácter precario y también parcial), mientras que los seis restantes trabajan en la universidad a tiempo completo bajo la figura de profesor ayudante doctor. Para la selección de los participantes se empleó un muestreo intencional (Suri, 2011). Los criterios de selección fueron: (a) que todos los docentes estuvieran trabajando en la universidad bajo la figura de profesor asociado o profesor ayudante doctor -las dos figuras más precarias del sistema universitario español-; (b) estar interesados en realizar carrera 
profesional dentro de la universidad; y (c) ser conocedores de los requisitos que establece el sistema nacional de acreditaciones y calidad de la evaluación docente para poder promocionar dentro del sistema universitario.

Debido a las diversas características y experiencia profesional de los participantes, el método de muestreo fomentó la elaboración de narrativas ricas en información relacionada con el propósito del estudio, una elevada calidad de las descripciones de los participantes, y una identificación de patrones profesionales significativos.

\section{Recogida de Datos}

Todas las entrevistas fueron realizadas por el autor del trabajo, quien se reunió durante el curso 2018/2019 con cada uno de los participantes del estudio durante un periodo de entre una y dos horas en un lugar de su elección (por ejemplo, el lugar de trabajo). El inicio de la conversación se centró en una serie de preguntas relacionadas con: (a) ¿qué es para ti ser docente novel?; (b) ¿qué entiendes por precariedad dentro del trabajo universitario?; (c) ¿cuál es tu plan para desarrollar una carrera profesional dentro de la universidad?; y (d) ¿qué implicaciones, a nivel personal y/o profesional, tiene para ti querer hacer carrera dentro de la universidad?

Al final del curso, el autor volvió a entrevistar a los participantes, esta vez centrándose en el periodo actual como forma de conocer lo que les había acontecido a lo largo del curso universitario y de conocer sus experiencias en todo ese tiempo. En esta segunda parte se utilizaron las siguientes preguntas como acicate para la narración verbal: (a) ¿qué es lo que más se valora de tu currículum vitae para trabajar en la universidad?; (b) ¿qué plan has trazado a lo largo del curso para hacer carrera en la universidad?; y (c) ¿a qué has renunciado a lo largo de este curso para favorecer tu carrera?

A continuación se inició un proceso de tres pasos con cada uno de los participantes como una forma de contar sus historias. El primer paso incluía la narración verbal (McMahon \& McGannon, 2019) de sus experiencias. En el segundo paso, el autor transcribió sus relatos verbales en una forma de historia escrita. La escritura de la historia fue elegida por el autor y los participantes en la convicción de que esta forma sería más atractiva para la audiencia que se comprometiera con ella. Una vez que las representaciones de la historia se construyeron en forma escrita, el autor sintetizó las 
principales ideas aportadas por los participantes en una sola historia. La historia se transmitió a cada uno de los participantes, para que pudieran aprobarla, añadirla o modificarla.

\section{Análisis Narrativo}

Los datos fueron analizados en términos narrativos. El análisis narrativo de las transcripciones se llevó a cabo a través de la interpretación de aquello que fue dicho (Riessman, 1993). Inicialmente, esto condujo a la identificación de los principales temas. Los temas surgidos en las diferentes entrevistas se agruparon en torno a diferentes categorías, tal y como se refleja en la tabla 1 (ver tabla 1). A partir de estas categorías se elaboró el relato. 
170 González-Calvo - El Agar.io Programado en la Enseñanza Superior

Tabla 1.

Categorías surgidas a partir de las entrevistas a los participantes.

EL DOCENTE NOVEL

\begin{tabular}{lll}
\hline \multicolumn{1}{c}{$\begin{array}{c}\text { Categorías de } \\
\text { análisis }\end{array}$} & Subtemas que aparecen & \multicolumn{1}{c}{ Temas de la entrevista } \\
\hline $\begin{array}{l}\text { (Auto)consideración } \\
\text { como docente novel }\end{array}$ & Sí-No & $\begin{array}{l}\text { ¿Por qué? } \\
\text { ¿Qué significa ser DN? } \\
\text { y precario }\end{array}$ \\
$\begin{array}{llll}\text { Características del DN } \\
\begin{array}{l}\text { Problemáticas del } \\
\text { docente novel } \\
\text { precario }\end{array}\end{array}$ & y & Cómo se manifiesta \\
\hline
\end{tabular}

LA CARRERA UNIVERSITARIA

\begin{tabular}{lll}
\hline \multicolumn{1}{c}{$\begin{array}{c}\text { Categorías de } \\
\text { análisis }\end{array}$} & Subtemas que aparecen & Temas de la entrevista \\
\hline $\begin{array}{l}\text { Las condiciones de } \\
\text { posibilidad para }\end{array}$ ¿Constituye preocupación una hacer & \\
configurar 'mi' & $\begin{array}{l}\text { 'carrera universitaria'? } \\
\text { futuro }\end{array}$ & $\begin{array}{l}\text { ¿Pensáis en ello?, ¿os } \\
\text { trazáis algún plan? }\end{array}$ \\
& La 'carrera universitaria' & $\begin{array}{l}\text { En qué consiste } \\
\text { Cómo se hace } \\
\end{array}$ \\
& & $\begin{array}{l}\text { Cuánto dura } \\
\text { Exigencias que conlleva }\end{array}$
\end{tabular}

continúa 
Tabla 1.

Categorías surgidas a partir de las entrevistas a los participantes

(continuación)

LA VIDA UNIVERSITARIA

Categorías de Subtemas que aparecen Temas de la entrevista análisis

\begin{tabular}{|c|c|c|}
\hline \multirow[t]{2}{*}{$\begin{array}{l}\text { Peso/papel de la } \\
\text { docencia }\end{array}$} & $\begin{array}{l}\text { Relevancia de la docencia } \\
\text { al } \\
\text { acreditarse, } \\
\text { carrera... }\end{array}$ & $\begin{array}{l}\text { El trabajo docente 'bien hecho', } \\
\text { ¿vale para algo? }\end{array}$ \\
\hline & $\begin{array}{l}\text { El tiempo (cuánto...) del } \\
\text { trabajo docente: } \\
\text { preparación, tutorías- } \\
\text { supervisión, "virtual"... } \\
\text { Peso de la docencia en } \\
\text { comparación } \\
\text { publicaciones } \\
\text { investigaciones }\end{array}$ & $\begin{array}{l}\text { ¿Se estimula-anima a hacer } \\
\text { bien-mejorar el trabajo } \\
\text { docente? }\end{array}$ \\
\hline \multirow[t]{2}{*}{$\begin{array}{l}\mathrm{La} \quad \text { (perversa) } \\
\text { dinámica }\end{array}$} & $\begin{array}{l}\text { Centra la atención en } \\
\text { formar } \\
\text { de...proyectos parte } \\
\text { investigación }\end{array}$ & \\
\hline & $\begin{array}{l}\text { Centra la atención en } \\
\text { formar parte de... grupos } \\
\text { o redes }\end{array}$ & $\begin{array}{l}\text { (La obligación de publicar } \\
\text { condiciona o incide...) }\end{array}$ \\
\hline
\end{tabular}

continúa 
172 González-Calvo - El Agar.io Programado en la Enseñanza Superior

Tabla 1.

Categorías surgidas a partir de las entrevistas a los participantes

(continuación)

LA VIDA UNIVERSITARIA

\begin{tabular}{|c|c|c|}
\hline $\begin{array}{l}\text { Categorías de } \\
\text { análisis }\end{array}$ & Subtemas que aparecen & Temas de la entrevista \\
\hline \multirow[t]{11}{*}{$\begin{array}{l}\mathrm{La} \quad \text { (perversa) } \\
\text { dinámica }\end{array}$} & $\begin{array}{l}\text { Centra la atención en... } \\
\text { publicar }\end{array}$ & $\begin{array}{l}\text { Los posibles objetos de estudio } \\
\text { (qué) }\end{array}$ \\
\hline & & $\begin{array}{l}\text { Los enfoques del estudio } \\
\text { (cómo) }\end{array}$ \\
\hline & & Sumisión a... \\
\hline & & Autorías falsas \\
\hline & & Auto-plagios \\
\hline & $\begin{array}{l}\text { Una particular forma de } \\
\text { auditoría cultural }\end{array}$ & $\begin{array}{l}\text { (cambios menores en un mismo } \\
\text { artículo) }\end{array}$ \\
\hline & & Círculo de citas \\
\hline & & Invención \\
\hline & & ¿Qué cuenta \\
\hline & & conocimiento? \\
\hline & & Tener más ISIs y proyectos \\
\hline
\end{tabular}

LA VERTIENTE PERSONAL

Categorías de Subtemas que aparecen Temas de la entrevista análisis

Configuración de la Yo soy mi C.V.

¿Cuál es la mejor manera de propia identidad Soy en función de mi hacer currículum vitae? puesto en la jerarquía

Procesos de Docencia: evitar adaptación, des- problemas y 'satisfacción empoderamiento y del cliente' interiorización de Publicar o morir dos reglas 
Tabla 1.

Categorías surgidas a partir de las entrevistas a los participantes (continuación)

LA VERTIENTE PERSONAL

\begin{tabular}{lrll}
\hline $\begin{array}{l}\text { Categorías } \\
\text { análisis }\end{array}$ & de & Subtemas que aparecen & Temas de la entrevista \\
\hline $\begin{array}{l}\text { ¿Sensación } \\
\text { riesgo o de } \\
\text { angustia? }\end{array}$ & cierta & $\begin{array}{l}\text { ¿Cuánto dura? } \\
\text { Necesidad de publicar } \\
\text { No quedarse atrás } \\
\text { Acreditación=evaluación }\end{array}$ & $\begin{array}{l}\text { Estabilidad-seguridad } \\
\text { Sueldo digno, ¿a qué edad? } \\
\text { Tentación del abandono, } \\
\text { pluriempleo... } \\
\text { Lógica de la Universidad: se } \\
\text { nutre de asociados por } \\
\text { rentabilidad económica. }\end{array}$ \\
$\begin{array}{l}\text { Repercusiones } \\
\text { físicas } \\
\text { psicológicas }\end{array}$ & ¿Novel y quemado? & Crisis profesional \\
\hline
\end{tabular}

DIMENSIÓN/CONTEXTO SOCIO-POLÍTICO

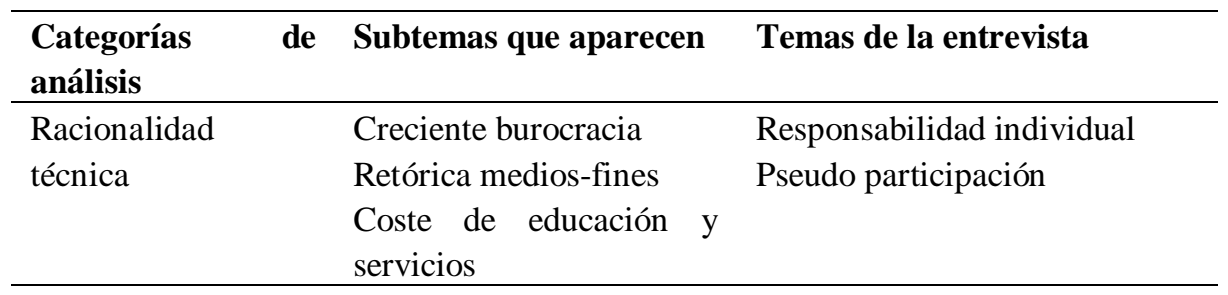

\section{¿ES DISTINTO PARA UN HOMBRE QUE PARA UNA MUJER?}

\begin{tabular}{lrll}
\hline $\begin{array}{l}\text { Categorías } \\
\text { análisis }\end{array}$ & de & Subtemas que aparecen & Temas de la entrevista \\
\hline $\begin{array}{l}\text { Ventajas } \\
\text { inconvenientes }\end{array}$ & y/o & $\begin{array}{l}\text { Renuncias a formar una } \\
\text { en }\end{array}$ & $\begin{array}{l}\text { ¿A qué tengo que renunciar? } \\
\text { torno al género }\end{array}$ \\
& $\begin{array}{l}\text { El papel reproductor de la encuentro? } \\
\text { mujer puesto en } \\
\text { entredicho }\end{array}$ & \\
& & \\
\end{tabular}


Luego, el autor emprendió una segunda ronda de análisis a través de un proceso de comparación constante de los textos a fin de establecer subtemas creíbles y fiables en cada categoría (Gubrium \& Holstein, 2009). Posteriormente, se seleccionaron citas pertinentes para ilustrar estos temas y subtemas con las que, finalmente, se elaboró el relato.

\section{Resultados y Discusión}

La siguiente narrativa recrea una historia que toma como protagonista a una mujer $^{3}$ que, trabajando a tiempo parcial como profesora en la universidad, pretende hacer carrera profesional dentro de ella. La línea argumental pone en primera línea los dilemas y debates de su elección, tanto personales como políticos, económicos, sociales y educativos. Creemos que se trata de una ficción que narra una situación asumible por gran parte de los profesionales que optan a un puesto dentro de la educación superior en un momento imbuido de recortes, precariedad e incertidumbres. A este respecto, en las conversaciones que hemos mantenido con ellos a partir del relato elaborado, manifiestan sentirse bien representados.

Nuestra narración combina dos tipos de texto. El primero de ellos, en formato cursiva, cuenta la historia que hemos elaborado en torno a un día normal en la vida de una profesora universitaria con una plaza precaria e inestable. El segundo tipo de texto, en letra normal, recoge las interpretaciones de la historia, cotejadas con la literatura científica. De este modo, queremos sugerir que esta narración tiene protagonistas diferentes y que podría ocurrir que sus relatos no fuesen coincidentes, realimentando el diálogo y abriendo nuevas perspectivas de las que queremos hacer partícipes a los lectores.

Cuando terminé de doctorarme en la universidad, la mayor parte de mis amigos y compañeros ya tenían un trabajo relativamente estable. Yo, de manera inocente, pensaba que en breve también tendría un trabajo fijo, bien remunerado y con mayor prestigio que muchos otros: profesora universitaria. Pero la recesión había calado hondo en el ámbito de la educación y, al igual que se culpa de todo al clima (desde las sequías hasta las inundaciones), el receso económico era responsable de todo. Un mal augurio que había soplado todas las buenas condiciones laborales igual que el viento arrastra el polvo del camino. Aun así, era optimista: mi futuro 
profesional estaba en el epicentro de la recesión pero en breve, cual ave Fénix, renacería la edad de oro de la universidad y, con ella, llegaría mi ansiado y prometedor futuro profesional. De esto hace más de diez años.

Es una tarde agradable, cálida, primaveral, la clase de tarde por la que tendría que estar agradecida de existir. El aire huele a humo, a flores, a elementos químicos, a soledad. Acabo de llegar a mi apartamento alquilado. Un oscuro pasillo, una pequeña cocina y una habitación todavía más oscura y pequeña. Mi universo se ha contraído hasta convertirse en este apartamento y el despacho de la universidad, mi cárcel y mi libertad. El pago del alquiler me cuesta la totalidad del sueldo de uno de mis dos trabajos precarios. Si tuviera menos sueños, menos inquietudes laborales o, quizá, un poco más de mundo, habría renunciado hace tiempo a seguir intentando desarrollar mi carrera profesional en la universidad. Pero sigo perseverando: soy una extraña que sabe demasiado bien cómo funcionan las cosas, que se siente vacía la mayor parte del tiempo y, aun así, alguien que renuncia a alcanzar su meta.

Después de comer rápido un plato precocinado (hace tiempo que todas las comidas tienen el mismo sabor insípido, pero no puedo perder tiempo en cocinar) me acuesto en la cama con la intención de descansar brevemente. Tumbada, vuelven mis pensamientos diarios sobre el momento en que mis ideales sobre lo que significa ser profesora universitaria cambiaron por otros tan sombríos en los que la competitividad, el estrés, la mayor asunción de riesgos (personales y profesionales) y la inseguridad serían mis compañeros de trabajo. Rondo los cuarenta años y todavía no he conseguido asentarme. Más aún: soy incapaz de trazar un plan de vida a corto plazo. De una forma u otra la inquietud, la vergüenza, el tedio, comienzan a asfixiarme. Si hasta hace poco mi cuerpo rugía de hambre y sed de ambiciones, ahora se conforma con sobrevivir día tras día. Me gustaría dormir y recuperar todo el sueño acumulado de los últimos meses. Pero no puedo perder tiempo durmiendo. Formo parte de esa mitad de la población que, a diferencia de la otra media, no toma pastillas para dormir, sino para mantenerse despierta.

A pesar de mi resistencia, consigo dormitar unas horas. Me levanto con la espalda dolorida y con la culpa por dejar que el sueño me cogiera por sorpresa. La noche fue hecha para descansar, y el día vuelve demasiado pronto. Solo el cuerpo duerme. Puede que esté llegando a mi límite. Me 
encuentro cansada, y no es el tipo de cansancio que puede arreglarse durmiendo un día entero en una cama cómoda. Es un cansancio que ha pasado a formar parte de mí. Mi cuerpo, mi mente, están adormecidas. A cambio de ese sueño reparador, decido darme una ducha que me ayude a sobrellevar el cansancio. Me observo en el espejo, mirando por encima de mi hombro para saber qué es lo que viene, en lugar de mirarme directamente a mí para saber qué es lo que hay. Si años atrás, al mirarme a los ojos, pensaba que brillaban con lo que solo podía ser inteligencia ahora el brillo, caso de tenerlo, es de melancolía. ¿Por qué lo que antes brillaba termina por volverse opaco? ¿Puede lo que se vuelve opaco volver a brillar? Debería ser capaz de mirarme al espejo, aunque no me guste lo que vea en él. Y no me refiero a mi físico; me refiero a tener la valentía de enfrentar la mirada con el yo que está atrapado detrás del cristal.

Veo varias llamadas perdidas de mi madre. Se las devolveré más tarde. Ahora no quiero escuchar otra vez los sermones propios de una madre: "hija, comes poco", "hija, trabajas demasiado", "hija, tienes que dormir más”. Voces sin cuerpo, suerte de aparato el teléfono. Al menos hace tiempo que no me insiste con que busque una pareja y me plantee tener hijos. Mis abuelos tuvieron cinco hijos; mis padres tuvieron dos; yo no tengo ninguno. Es la misma secuencia que veo repetirse alrededor: cuatro-cinco, dos, uno o ninguno. En varias ocasiones me he tenido que enfrentar al dilema de elegir entre una carrera profesional o una vida más plena. “¿Qué puede haber más triste que no tener hijos?" me han dicho en varias ocasiones. Es posible que sea egoísta, mezquino e, incluso, antinatural; pero es mi elección. Además, mis padres siempre han trabajado y luchado durante años con la convicción de que me espera un futuro mejor que el que ellos tuvieron. Me han inculcado esa idea, lo que me lleva a sentirme menos preparada, si cabe, para un futuro inhóspito en el que las puertas siempre parecen estar cerradas. ¿Cómo podría yo siquiera plantearme ser madre con el futuro tan poco acogedor que se presenta ante mí?

Decido ponerme a trabajar, a actualizar mi currículum e introducir mis méritos académicos e investigadores en la aplicación informática para solicitar mi acreditación como profesora ayudante doctora, la segunda figura más precaria del mundo universitario. No es solo que las figuras de asociada y ayudante sean las más bajas en la jerarquía: es que todo el entramado universitario se encarga de recordar continuamente a quienes 
ocupamos estas plazas que nuestra labor es menos relevante que la de aquellos que se sitúan en escalafones superiores. En mi entorno se da la paradoja de que personas más bajas en la jerarquía tienen más currículum que en las altas esferas. Sin embargo, eso importa poco a la hora de conseguir financiación para congresos, becas de investigación y similares.

A medida que voy cumplimentando los datos me asalta la siguiente duda: ¿quién soy yo? A pesar de todos los méritos académicos que voy atesorando, todos ellos a costa de trabajar un tiempo extra, robando hasta los instantes al tiempo familiar, de ocio, de descanso, de vida, no lo tengo claro. Mi vida personal se ha fundido completamente con la profesional, de modo que ¿yo soy mi currículum vitae?, ¿o es más bien mi currículum vitae el que va identificándose poco a poco con mi personalidad? Más aún: ¿qué o quién era antes de lo que soy ahora?, y ¿qué ocurrió con aquello que era al convertirme en lo que ahora soy? Tantas dudas que me hacen sentir vacía de cabeza a pies. Prefiero entretener mis pensamientos con la aplicación informática.

Caigo en la cuenta de que en los últimos meses he incrementado mi perfil investigador y he conseguido publicar varios artículos. Ha sido a costa de lo que de verdad me gusta de la profesión: la docencia. Pero tengo que ser práctica: dar bien las clases consume tiempo y muchas energías, y su peso es irrelevante a la hora de hacer carrera. Al menos ya voy teniendo claro qué es lo que se espera de mí: que sea capaz de publicar en revistas que ocupen los primeros cuartiles de las bases de datos JCR y SJR ${ }^{4}$ Este requisito taylorista que modifica profundamente la labor docente, este instrumento político actual de dominación sobre el trabajo universitario, esta perversa dinámica de publicar o perecer, es mi leitmotiv.

Entrar en esta dinámica me genera malestar e irritación. Por momentos, aborrezco el instante en que decidí seguir este camino profesional. Me siento sola en el proceso, me tientan las ganas de abandonar y a menudo sueño despierta que encuentro un trabajo, un sueldo y una estabilidad en otros lugares. Todavía soy joven, llevo relativamente poco tiempo trabajando aquí y, no obstante, ya estoy quemada. No sé cuándo fue la última vez que me sentí libre y desocupada; ahora siempre me ronda en la cabeza la idea de ¿qué será lo próximo que publique?, ¿qué debo hacer para enriquecer mi currículum?, ¿cuáles serán las nuevas exigencias para seguir haciendo carrera en la universidad? Se me parte el alma también saber que la 
burocracia es mi día a día en la profesión, que no se puede subir de escalafón ni obtener una recompensa, por mínima que sea, si no es mediante procesos de control, verificación y certificación infinitos.

Por otro lado pienso qué puede suponer no acreditarme. Desde una perspectiva general, no acreditarme no es más que un ensayo para perderlo todo, el comienzo del fin de mi carrera. ¿A quién podré culpar si llega ese momento? Sé que tardarán en contestar unos meses antes de evaluarme positiva o negativamente. Suena extravagante, casi grotesco, esperar que con esa notificación va a llegar mi hora, una notificación que pondrá en orden mi vida. De modo que pongo empeño en cumplimentar la aplicación: casi diría que se trata de una tabla en la que en un lado aparecen "el haber" $y$, en la otra, "el debe", siendo siempre la segunda mucho mayor que la primera. Y es que es perpetua la sensación de que, a pesar de intentarlo todo y poderlo todo, nada es suficiente.

En la universidad es común que nos eduquen (¿o quizá nos adiestran?) para creer que el trabajo es el remedio que todo lo cura o, cuando menos, un recurso para aliviar todos los males y aflicciones de nuestras vidas particulares y en común, si no de las actuales, sí de las futuras. Al mismo tiempo existe una sensación de temor a no cumplir las expectativas, más aún en un contexto en el que el "otro" (cualquier "otro") es una amenaza, ya sea en forma de compañero de departamento, de exigencias del sistema o bien en forma todavía no revelada (y, por ese motivo, más aterradora). Es una época en la que tenemos que culparnos a nosotros mismos de todos los problemas imaginables. Hace tiempo que sé que la cooperación, la empatía y la solidaridad son valores propios de personas ingenuas, insensatas, sin metas en la vida. La esperanza no entiende de frivolidades: se trata de estar en un nivel superior a los demás y, una vez alcanzas esos niveles, no pararse a mirar hacia abajo. Si existió un tiempo en el que dentro de la universidad los profesores podían mejorar sus condiciones trabajando colectivamente, ahora los que estamos aquí entendemos que nuestra única opción es protegernos a nosotros mismos. Ahora somos competidores de todos los demás, y ya ni siquiera nos tomamos la molestia de disimularlo. Tanto a mí como a mis compañeros nos enseñan a depender de nosotros mismos y estar preparados en cualquier momento para cuando sobrevengan tiempos revueltos. Nos enseñan, al fin y al cabo, a (auto)culparnos de todos los problemas. 
Si quiero montar en el caballo desbocado que es la universidad, no me queda más remedio que tratar de cumplir con las exigencias del sistema en la medida de mis posibilidades. Mis posibilidades, que no son tantas como quisiera ni dependen tanto de mí como de mis circunstancias. El sistema neoliberal en el que se mueve la universidad, que pienso si servirá para algo más que para incrementar los gastos burocráticos y el sufrimiento del personal, expulsa a aquellos que, teniendo talento, no tienen posibilidades de financiarse una carrera ad hoc. Termina por convertirse mi vida en algo impersonal, una fuente inagotable de cansancio, ansiedad y desolación en la que la estabilidad, el orden, el control y el saberte dueña de tus propias decisiones hace tiempo que se esfumaron. Echo de menos ser joven, esos años en los que uno no discute su vida, la vive. He perdido parte de los motivos para luchar; ya hace tiempo que no disfruto de las caricias del sol en la cara, de escuchar música, leer una novela, pasear por el campo. ¿Qué me ofrece a cambio la universidad? Sin saberlo sigo adelante, quizá solo por el hecho de que no me gustaría pensar, en el futuro, que me faltó voluntad de sacrificio justo cuando estaba a punto de conseguirlo, desde esa lógica que me lleva a creer que yo, entre tantos otros, "soy yo la elegida”. Mas, en el fondo de mi cabeza late la idea de que, en ocasiones, un abandono a tiempo es una victoria.

Hace tiempo que la imagen que se tiene del profesor universitario ha cambiado. Ya no es tan común la idea de persona curiosa, disciplinada, con ansias de saber y con aura de admiración. Una profesión que es una forma de vida; una que no permite vivir en la opulencia económica, pero sí la suficiente autonomía y conciencia crítica como para explorar las fronteras del conocimiento y compartirlas con su alumnado. Eternos estudiosos, exploradores del conocimiento y maestros docentes. Pero hace años se viene observando una deriva del oficio que está minando, lenta pero incansablemente, la motivación de quienes escogen esta labor.

En el año 2009, la universidad española se integró en la inclusión del llamado Plan Bolonia, con la intención de adecuarse a las pretensiones europeas en relación a la estandarización de criterios y exigencias en el contexto universitario (Menéndez, 2014). Con esta medida, iniciada en 1998 con la firma de las bases de la Declaración de la Sorbona, se garantizaba la plena consolidación del Espacio Europeo de Enseñanza Superior. 
Entre una mala aplicación del Plan Bolonia y la tremenda recesión económica que la universidad pública lleva experimentando desde la crisis económica de 2011, la proporción de profesorado en situación precaria y con escasa retribución económica ha ido en aumento. Los profesores asociados, el último eslabón de la cadena de precariedad de la universidad pública, son los responsables de suplir las carencias que muestra el sistema universitario. Así, en el curso 2016/2017 el 23,6\% de la plantilla en las universidades era profesorado asociado o "profesores pobres", en notable aumento en los últimos años.

En España, la figura de profesor asociado surge con la intención de que profesionales de un determinado campo puedan compartir su experiencia con futuros profesionales (e.g., médicos, maestros y/o arquitectos formando, desde su perspectiva profesional, a futuros médicos, maestros $\mathrm{y} / \mathrm{o}$ arquitectos). Con la crisis económica, esta figura se ha pervertido y la universidad se ha lucrado de esta figura para suplir, en la medida de lo posible, la baja tasa de reposición del profesorado a que han obligado los diferentes partidos políticos. De esta manera, es fácil encontrarse con profesores que trabajan jornadas enteras en la universidad llevando a cabo labores de docencia, investigación y gestión por sueldos que rondan los 300600 euros. En el caso de la segunda figura universitaria con mayor inestabilidad (profesor ayudante-doctor), la situación no es mucho mejor. Son dos figuras laborales muy eficaces para obtener trabajo a bajo coste y en las que la precariedad cobra un protagonismo excesivo (ninguna de las dos proporciona una estabilidad asegurada, si bien los participantes consideran la figura de ayudante-doctor como más estable que la de asociado). Puesto que los profesores, si quieren promocionar dentro de la universidad, tienen que acumular experiencia docente, no les queda más remedio que optar por plazas muy mal retribuidas.

Algo similar sucede con la investigación: colaboran en proyectos de innovación e investigación de manera gratuita, publican artículos y actúan como revisores de artículos sin ninguna remuneración económica. Al profesor en precario no le queda otra opción que adaptarse a las continuas y variables demandas del sistema, que suponen una enorme dedicación laboral e, incluso, la combinación de diferentes trabajos en condiciones de precariedad (Lorenz, 2012). Todo ello al tiempo que se incrementan las exigencias de rendimiento, de competitividad laboral y las dificultades para 
poder conciliar la vida personal con la profesional (algo más evidente, como hemos visto, en el caso de las mujeres). La figura del profesor universitario queda así sometida a los imperativos del sistema, la precarización de sus condiciones laborales y la pérdida de prestigio y estatus simbólicos de que antes gozaba.

Entendiendo la dinámica del discurso neoliberal estaremos en condiciones de comprender los fenómenos vinculados con la precariedad, la ansiedad y el desencanto en términos de comportamientos individuales, de oportunidades y esfuerzos personales (o, por el contrario, la falta de esfuerzo). Se reconducen los problemas sociales (recortes económicos, excesiva carga burocrática para el profesorado, sueldos mínimos) a los talentos y capacidades de cada persona: cada uno tiene lo que se merece, bajo el falso precepto de "si te esfuerzas, lo conseguirás". El docente no tiene otra salida que aportar continuos rendimientos, lo que hará que pueda seguir formando parte del sistema o, por el contrario, fracase y se quede fuera del mismo. Esta situación conduce a que el profesorado universitario, en lugar de considerar su carrera profesional como una labor llena de sentido, plena y segura, la entienda como una utopía inalcanzable dada la situación social y económica que rodea a la enseñanza superior. Las metas que se (auto)imponen son lejanas, casi inalcanzables, lo que les lleva a pensar que no es posible un mundo mejor que el que ahora tienen. Al contrario, parece que las perspectivas futuras, tal y como están las cosas, serán cada vez más sombrías. Como explica Zafra (2017), el contexto de los profesionales precarios está definido por trabajos y prácticas temporales; sujetos envueltos en inestabilidad y travestidos de un entusiasmo fingido que se emplea para aumentar su productividad. La precariedad, al igual que el entusiasmo, se ha convertido en un arma para explotar a los trabajadores temporales y sofocar la disidencia, manteniéndolos preocupados por el temor de perder sus empleos (Giroux, 2014). Esta precariedad es política, social y económicamente amenazante, ya que genera enojo, ansiedad y alienación (Standing, 2011).

A todo ello se le suma que el sistema neoliberal fomenta una creencia desenfrenada en los valores del mercado, el individualismo radical y la competencia desenfrenada, por lo que desalienta las nociones de público, solidaridad y cuidado de los demás (Giroux, 2014). En el mundo neoliberal, el yo ágil y mercantil es valorado y atesorado, ya que puede encarnar los 
deseos corporativos y ser capturado por el discurso neoliberal (Gillies, 2011). La condición necesaria de la "agilidad", como precursora del éxito en el mundo neoliberal, se hace eco de lo que Bauman (2000) denominó "liquidez", como una forma de caracterizar la relación que los individuos tienen con la sociedad actual. Nuestras subjetividades están en continuo cambio y, como fluidos, no somos capaces de mantenemos en ninguna forma por mucho tiempo y necesitamos estar constantemente preparados (y propensos) al cambio (Bauman, 2000). De no ser así, nuestras subjetividades serán fuente de frustración.

Por otra parte, la continua evaluación del profesorado universitario, más aún de aquellos que trabajan en condiciones precarias, da lugar a una trama de autodisciplina colectiva y de vigilancia recíproca (Kerfoot \& Knights, 1995) que canaliza la mayor parte de la actividad política y participativa del empleado en la institución hacia una exigencia de mayores y mejores sistemas de rendimiento individual. Se trata, como afirma Noll (2019), de una nueva cultura organizativa de la calidad, en la que las exigencias de producción y calidad son asumidas y promovidas por el propio trabajador en sus interacciones con el resto y cuyas resistencias a su aplicación pueden ser vistas como deslealtades a la organización (Tuckman, 1995). Un claro ejemplo es la desmesurada producción científica que exige el sistema de acceso a la profesión: puesto que la Agencia Nacional de Evaluación de la Calidad y Acreditación exige al profesorado la publicación en bases de datos JCR y SJR (y, preferiblemente, en revistas situadas en los primeros cuartiles), los docentes/investigadores han tenido que renunciar a tareas de investigación y difusión que no correspondan a ese objetivo. Como señala Noll (2019), no solo dejan de realizarse investigaciones social y científicamente útiles sino que, también, desaparecen múltiples actividades de indudable valor social como asesorar a empresas y sindicatos, participar en medios de comunicación y participar en congresos y reuniones científicas, entre otros.

Así, las personas que luchan por hacer carrera en la universidad consideran que el sistema promueve una pedagogía del desamparo, fatiga y soledad (González-Calvo, 2020). El mismo mecanismo que se encuadra dentro del precepto de "no pienses demasiado en nada; piensa solamente en ti mismo". Está por ver si el engranaje de la carrera profesional universitaria no termina por obligar a cambiarse a uno mismo, aceptando de forma acrítica 
y sometiéndose a las condiciones de explotación y alienación del entorno socioeconómico y cultural.

\section{Conclusiones}

Son muchas las condiciones que se le exigen a un profesor novel dentro de la enseñanza superior -calidad, excelencia, acreditaciones, estancias de investigación, entre otras- que son difíciles de cumplir e, incluso, contradictorias con la situación social y económica que caracteriza a las instituciones universitarias de hoy día. Pareciera que la esperanza se esté privatizando (Bauman, 2017), trasladando las aspiraciones y las responsabilidades que corresponden a la universidad al plano individual. Quizá hubo un tiempo en que trabajar en la universidad fuera sinónimo de motivación, libertad, ilusión; ahora, quienes trabajan en ella comprenden que el estrés, la ansiedad y el protegerse a uno mismo son los criterios que mandan. No terminan de encontrar un sentido a su vida profesional (y, por extensión, personal); su labor requiere ser alguien ferozmente competitivo, codicioso, que ve en cualquier otro individuo un rival al que vencer y que lo lleva a vivir en un estado de inquieto y permanentemente insatisfecho deseo (Lasch, 1979). La idea subyacente del nuevo modelo universitario la proporciona el homo economicus egoísta, acrítico, flexible, emprendedor; un tipo humano orientado por la racionalidad del beneficio y que, como empresario de sí mismo, considera irracional cualquier gesto de altruismo o fraternidad (Fernández Liria et al., 2017).

Si la universidad continúa rigiéndose exclusivamente por las leyes mercantiles y neoliberalistas se deshumanizará cada vez más, generando convulsiones sociales y verdaderos problemas personales y profesionales entre su profesorado. Por esa razón, es imprescindible que las políticas educativas de la educación superior vayan acompañadas de otras políticas vitales que siembren solidaridad y civismo; sin ella surgen unas masas inseguras, movidas por el miedo y sin estructuras estables en el tiempo (Han, 2018).

Es importante tener en cuenta que los profesionales que trabajan en la universidad merecen ser considerados un fin en sí mismo, y no un instrumento para el beneficio capitalista y mercantilista que en nada ayuda a promover justicia, bienestar y una vida mejor, propósitos que pretendía la 
universidad humanista de no hace demasiado tiempo. Si las universidades siguen apostando por las ganancias y no por la conciencia, terminarán convirtiéndose en entidades opresoras que llevarán a que el profesorado caiga en la resignación, el fatalismo y el conformismo. Por ello, y aun sabiendo que las personas no somos parte de un Agar.io, es posible que una especie de teleología lleve al profesorado universitario a ser devorado, invadido, desechado, consumido, terrible metáfora deshumanizadora de la universidad. Para evitar encabezar la comitiva fúnebre del "game over" profesional se hace necesario, más que nunca, buscar alternativas que inviten a pensar, dialogar y actuar colectivamente para reconstruir un "nosotros" que haga frente al insensible e individualista sistema neoliberal universitario.

\section{Notas}

${ }^{1}$ Datos y estadísticas del profesorado de las universidades españolas [https://www.epdata.es/datos/profesores-investigadores-resto-personal-docente-universidadespana-datos-estadisticas/412] [consultado el 15.06.2020].

${ }^{2}$ Extraído de www.epdata.es [consultado el 15.06.2020]

${ }^{3} \mathrm{Si}$ se ha elegido como protagonista de la historia a una mujer es porque, del análisis de los resultados, se refleja que son ellas quienes sienten mayor presión en torno al sistema, en aspectos tales como la maternidad (se ven abocadas a elegir entre su carrera profesional o ser madres) y por la culpabilidad que sienten creyéndose responsables del abandono de una u otra faceta.

${ }^{4}$ La página web de la Fundación Española para la Ciencia y la Tecnología orienta hasta un $90 \%$ de sus contenidos a la recomendación de fórmulas y maneras de mejorar el índice de impacto de los investigadores españoles y sus posibilidades de publicación en revistas indexadas en bases de datos JCR y SJR. [https://www.recursoscientificos.fecyt.es/inicio] [consultado el 19.02.2020].

${ }^{5}$ En palabras del Presidente de la Conferencia de Rectores Españoles, Roberto Fernández. [https://www.granadahoy.com/granada/mantener-Universidad-espanola-profesorespobres_0_1225677867.html] [consultado el 25.2.2020].

\section{References}

Apple, M. (2006). Educating the "right" way: markets, standards, God and inequality. Taylor and Francis.

Archer, L. (2008). The new neoliberal subjects? Young/er academics' constructions of professional identity. Journal of Education Policy, 23(3), 265-285. https://doi.org/10.1080/02680930701754047 
Bauman, Z. (2000). Liquid modernity. Polity Press.

Bauman, Z. (2017). Retrotopia. Polity Press.

Clarke, J. (2008). Power, politics, and places: What's not neo-liberal? European Journal of Anthropology, 51, 158-160. https://search.proquest.com/openview/730d527161c29dd3850e589b ec172b40/1?pq-origsite $=$ gscholar $\&$ cbl $=54515$

Chiapello, E. (2017). Critical accounting research and neoliberalism. Critical Perspectives on Accounting, 43, 47-64. https://doi.org/10.1016/j.cpa.2016.09.002

Chomsky, N. (2013). El trabajo académico, el asalto neoliberal a las universidades y cómo debería ser la educación superior. Bajo el Volcán, 13(21), 121-134. https://www.redalyc.org/articulo.oa?id=28640302006

Deem, R., Hillyard, S., \& Reed, M. (2007). Knowledge, Higher Education and the new managerialism. The changing management of UK Universities. Oxford University Press.

Díez Gutiérrez, E. (2018). Neoliberalismo educativo: educando al nuevo sujeto neoliberal. Octaedro.

Fernández Liria, C., García Fernández, O., \& Galindo Ferrández, E. (2017). Escuela o barbarie. Entre el neoliberalismo salvaje y el delirio de la izquierda. Akal.

Frank, A. (2004). Asking the right question about pain: Narrative and phronesis. Literature and Medicine, 23(2), 209-225. http://dx.doi.org/10.1353/lm.2005.0003

Frank, A. (2010). Letting stories breathe: A socio-narratology. The University of Chicago Press.

Gillies, D. (2011). Agile bodies: a new perspective on neoliberal governance. Journal of Education Policy, 26(2), 207-223. https://doi.org/10.1080/02680939.2010.508177

Giroux, H. (2014). Public intellectuals against the neoliberal university. En N. Denzin \& M. Giardina (Eds.), Qualitative inquiry outside the academy (pp. 35-60). Left Coast.

González-Calvo, G. (2020). Riesgos, entusiasmos e incertidumbres en torno a la carrera profesional universitaria: el nudo gordiano de la investigación en España. Márgenes Revista de Educación de la Universidad de Málaga. https://doi.org/10.24310/mgnmar.v1i1.6797 
González-Calvo, G., \& Arias-Carballal, M. (2018). Effects from audit culture and neoliberalism on university teaching: an autoethnographic perspective. Ethnography and Education, 13(4), 413-427. https://doi.org/10.1080/17457823.2017.1347885

Gubrium, J. F., \& Holstein, J. A. (2009). Analyzing narrative reality. Sage. Han, B. C. (2015). The burnout society. Stanford Briefs.

Han, B. C. (2018). The expulsion of the other: Society, perception and communication today. Polity Press.

Kerfoot, D., \& Knights, D. (1995). Empowering the "quality worker". En A. Wilkinson \& H. Willmott (Eds.), Making quality critical (pp. 219239). Routledge.

Lasch, C. (1979). The culture of narcissism: American life in an age of diminishing expectations. Warner Books.

Laval, C., \& Dardot, P. (2018). El ser neoliberal. Gedisa.

Lorenz, C. (2012). If you're so smart, why are you under surveillance? Universities, neoliberalism, and new public management. Critical Inquiry, 38(3), 599-629. https://doi.org/10.1086/664553

Luna, V. M. I. (2015). From neoliberalism to possible alternatives. Economía Informa, 395, 35-49. https://doi.org/10.1016/j.ecin.2015.10.004

McMahon, J., \& McGannon, K. R. (2019). 'I hurt myself because it sometimes helps': former athletes' embodied emotion responses to abuse using self-injury. Sport, Education and Society, 1-14. https://doi.org/10.1080/13573322.2019.1702940

Menéndez, I. (2014). El Espacio Europeo de Educación Superior en España: incorporación de contenidos y metodologías de género en comunicación. Revista Internacional de Comunicación y Desarrollo, 1, 23-34. https://dialnet.unirioja.es/servlet/articulo? codigo $=4981123$

Noll, H. (2019). It's Taylorism, stupid! On the new scientific organization of research and teaching in the Spanish University. Sociología del Trabajo, 95, 1-18. https://doi.org/10.5209/stra.66436

Riessman, C. K. (1993). Narrative analysis. Sage.

Riessman, C. K. (2008). Narrative methods for the human sciences. Sage.

Saidel, M. (2016). La fábrica de la subjetividad neoliberal: del empresario de sí al hombre endeudado. Pléyade. Revista de Humanidades y Ciencias Sociales, 17, 131-154. https://redib.org/Record/oai_articulo1320016la-f\%C3\% A1brica-de-la-subjetividad-neoliberal-del-empresario-des\%C3\%AD-al-hombre-endeudado 
Smith, B., \& Sparkes, A. (2009). Narrative inquiry in sport and exercise psychology: What can it mean, why might we do it? Psychology of Sport and Exercise, 10, 1-11. https://doi.org/10.1016/j.psychsport.2008.01.004

Standing, G. (2011). The precariat: The new dangerous class. Bloomsbury Academic.

Suri, H. (2011). Purposeful sampling in qualitative research synthesis. Qualitative Research Journal, 11(2), 63-75. https://doi.org/10.3316/QRJ1102063

Tuckman, A. (1995). Ideology, qualitity and TQM. En A. Wilkinson \& H. Willmott (Eds.), Making quality critical (pp. 54-81). Routledge.

Zafra, R. (2017). El entusiasmo. Precariedad y trabajo creativo en la era digital. Anagrama.

Gustavo González-Calvo is Partial Time Lecturer of the Department of Didactics of Musical, Artistic and Corporal Expression at University of Valladolid, Spain.

Contact Address: Gustavo González Calvo, University of Valladolid, Faculty of Palencia, Department of of Didactics of Musical, Artistic and Corporal Expression, Avenida de Madrid, 44, 34004, Palencia, Spain. Email: gustavo.gonzalez@uva.es 